\title{
Integração ensino-serviço-gestão na saúde bucal em Santa Maria e região: relato de experiência em estágio curricular acadêmico e Residência Multiprofissional
}

\author{
Caroline Weber*; Maria Laura Fagundes**; Anderson Tambara*; Emir Dirlan**; Alessandra \\ Beltrame $^{* * * ;}$ Ariane Krassman ${ }^{* * *}$; Glauber Oliveira ${ }^{* * * *}$; Patrícia Campagnol*****; Beatriz \\ Unfer******; Luísa Helena do Nascimento Torres******; Jessye Melgarejo do Amaral Giordani ${ }^{* * * * * * *}$
}

* Residente do Programa de Residência Multiprofissional Integrada em Sistema Público de Saúde, Ênfase Gestão e Planejamento, UFSM

** Residente do Programa de Residência Multiprofissional Integrada em Sistema Público de Saúde, Ênfase Saúde da Família, UFSM

*** Cirurgiã-Dentista da Estratégia de Saúde da Família, Prefeitura Municipal de Santa Maria

**** Coordenadora da Política Municipal de Saúde Bucal, Prefeitura Municipal de Santa Maria

***** Coordenador Regional de Saúde Bucal, Secretaria Estadual de Saúde do Rio Grande do Sul, $4^{\text {a }}$ Coordenadoria Regional de Saúde

****** Docente do Curso de Odontologia, UFSM

Recebido em 21/07/2017. Aprovado em 03/10/2017.

\begin{abstract}
RESUMO
Os autores descrevem as experiências de integração ensino-serviço-gestão entre a Universidade Federal de Santa Maria, a Prefeitura Municipal de Santa Maria e a $4^{a}$ Coordenadoria Regional de Saúde do Rio Grande do Sul. O objetivo é apontar as principais ações planejadas e executadas por docentes, gestores, residentes e estudantes de graduação destas três instâncias. O envolvimento dos estudantes de graduação nas atividades de estágio obrigatório é avaliado por meio da análise dos relatórios e as experiências de formação em serviço dos residentes da Residência Multiprofissional por meio de registro das atividades realizados nos serviços públicos de saúde do município. Aborda-se os avanços alcançados na implementação das ações, as dificuldades e as potencialidades encontradas na sua articulação, além da importância dos espaços de integração destas instâncias como ambientes privilegiados na formação dos cirurgiões-dentistas e dos estagiários da Odontologia. O estreitamento das relações entre a Residência Multiprofissional em Saúde e a gestão municipal e regional permitiu a concretização de espaços de interlocução entre profissionais em formação, docentes, gestores e usuários do serviço, proporcionando a reflexão sobre o processo de produção do cuidado, bem como a corresponsabilização pela formação profissional e pela qualidade do serviço de saúde.
\end{abstract}

Descritores: Serviços de Integração Docente-Assistencial. Serviços de Saúde. Gestão de Serviços de Saúde. 


\section{INTRODUÇÃO}

O contexto histórico do surgimento da Odontologia é marcado pela individualidade, pela monopolização do saber, pelo foco na doença e na técnica e por ser considerada uma especialidade médica ${ }^{1}$. Pode-se compreender a estruturação da Odontologia a partir de dois aspectos. Primeiro, como segmento da Área da Saúde onde, juntamente com as outras disciplinas, contribuiu para a fragmentação do sujeito, influenciada pelos relatórios Flexner e Gies, que inspiraram inclusive a fragmentação dos currículos. Isto impossibilitou uma compreensão mais abrangente do saber e resultou no modelo tradicional e não resolutivo de educação no Ensino Superior Brasileiro, que forma profissionais muitas vezes com saberes que se restringem às técnicas e que são distantes e não compatíveis com muitas das demandas da sociedade ${ }^{2}$.

Quanto ao outro aspecto, esta mesma Odontologia falhou em tentar se relacionar com as outras clínicas e acabou por ignorar os problemas sociais, sendo incapaz de identificálos como sua responsabilidade, não se comprometendo com a saúde das pessoas e das coletividades, o que se pode inferir como reflexo da influência e do poder da lógica do mercado ${ }^{3}$. Ambos aspectos se voltam ao mesmo ponto: o comprometimento de como se trabalha e se pesquisa na Odontologia; de como se pensa o corpo, isolando-o da boca, ignorando as questões que interessam ao paciente que são relacionadas ao acesso, a efetividade e a qualidade dos serviços odontológicos ${ }^{3}$.

Quando há o esgotamento de um modelo em função da perda de confiança em seu conhecimento estrutural há o rompimento de um paradigma sendo substituído por outro modelo predominante $^{4}$. As diretrizes curriculares nos cursos da saúde enfatizam a necessidade da vivência no serviço, da aproximação e da sensibilização dos estudantes em formação para o conhecimento do contexto social no Brasil, bem como a identificação de suas necessidades, carências e potencialidades.

É necessário formar cidadãos aptos a enfrentar os problemas da atualidade e responder às demandas de saúde da população, levando em consideração suas características culturais, sociais, demográficas, políticas e econômicas ${ }^{5}$. Este desafio tem por objetivo não somente formar trabalhadores com perfil voltado para as especificidades do Sistema de Saúde brasileiro, mas também transformar o mundo do trabalho e seus profissionais por meio da reflexão crítica quanto à sua prática, criando novos sentidos para a mesma.

A integração ensino-serviço no Sistema Único de Saúde (SUS) envolve estudantes, professores, trabalhadores e gestores dos serviços de saúde em um processo coletivo com o objetivo de qualificar a formação e o trabalho profissional para a atenção à saúde ${ }^{6}$. Neste contexto, a integralidade e a interdisciplinaridade são consideradas eixos norteadores da formação, pois tem a capacidade tanto de produzir relações, sentidos, significados e práticas de cuidados dignas e éticas, quanto de superar a fragmentação do trabalho para efetivar o cuidado $^{5}$, pois a integração destes setores tem a proposta de diminuir a distância entre teoria e prática e tem foco no usuário ${ }^{6}$.

Para haver a consolidação da integração ensino-serviço, esta precisa ser agregada aos currículos das IES - Instituições de Ensino Superior - com a rotina dos sistemas de saúde ${ }^{7}$. Assim, este artigo apresenta um relato de experiência, de caráter descritivo, da integração ensino-serviço-gestão entre o núcleo da Odontologia na Residência Multiprofissional Integrada em Sistema Público de Saúde da Universidade Federal de Santa Maria (UFSM), as disciplinas de Odontologia em Saúde Coletiva 
(OSC) do Curso de Odontologia da UFSM, a Coordenação da Política de Saúde Bucal (CPSB) da cidade de Santa Maria e a $4^{\text {a }}$ Coordenadoria Regional de Saúde do Rio Grande do Sul (4 CRS). Tem por objetivo descrever as principais ações planejadas e executadas em conjunto visando fomentar a transformação do processo de formação profissional na área da saúde e a melhoria do processo de gestão das políticas, a fim de efetivar os princípios e diretrizes do SUS. Ainda, aborda os avanços alcançados na implementação dos projetos e as dificuldades e potencialidades encontradas na sua articulação.

\section{RELATO DA EXPERIÊNCIA}

O curso de Odontologia da UFSM iniciou a integração com os serviços públicos de saúde em 2009, por meio dos Estágios Curriculares Obrigatórios. No mesmo ano foi criada a Residência Multiprofissional Integrada em Sistema Público de Saúde da instituição, inserindo residentes na Atenção Básica/Estratégia de Saúde da Família e, posteriormente, em 2015, na Vigilância em Saúde/Gestão e Planejamento. O aspecto fundamental para o fortalecimento das relações entre as instituições de ensino e serviço foi a disposição de professores, estudantes e profissionais em fomentar transformações na formação e nos serviços de saúde, realizando a aproximação dos diferentes sujeitos, buscando o cumprimento da responsabilidade social da profissão e entendendo o papel formador do SUS e para o SUS no atendimento às necessidades de saúde da população.

Os estágios curriculares obrigatórios são cumpridos na Disciplina de Estágio em Serviços Públicos de Saúde, realizados pelos acadêmicos de graduação no último ano do curso, com carga horária de 240 horas. Os campos de estágio compreendem a rede municipal de saúde, na sua maioria, e alguns serviços de saúde da UFSM e do Exército e Aeronáutica. Os preceptores são cirurgiões-dentistas destes serviços e os professores da disciplina de Odontologia em Saúde Coletiva atuam como supervisores.

$$
\text { A residência multiprofissional }
$$
corresponde a um curso de pós-graduação lato sensu, com duração de dois anos e carga horária de 60 horas semanais, divididas entre atividades teóricas, teórico-práticas e práticas. Além dos encontros semanais de preceptoria de núcleo, são realizadas atividades teórico-práticas que envolvem tutores de núcleo (professores da UFSM) e residentes. Bimestralmente acontecem encontros envolvendo também preceptores de campo e núcleo (coordenadores municipal e regional, e cirurgiões dentistas da atenção básica) onde são discutidos os processos de trabalho e referenciais teóricos importantes para a prática.

\section{Estágio Curricular Acadêmico}

Quando chegam ao campo de estágio da atenção básica, os estudantes de Odontologia da UFSM se deparam com a dificuldade de entender o funcionamento destes serviços, apresentando, por vezes, ideias e conceitos equivocados sobre o sistema. Ficam surpresos quando encontram ambientes e consultórios limpos e organizados, com materiais de boa qualidade e em algumas unidades básicas, uma equipe integrada e trabalhando de forma multiprofissional. Pontos negativos também são relatados pelos estudantes como, por exemplo, a falta de materiais para isolamento absoluto e gaze, materiais de baixa qualidade como revelador e fixador de radiografias e a demora demasiada no retorno de algum equipamento quando esse vai para conserto, como caneta de baixa rotação e aparelho de raios-X. Os estudantes, sob a supervisão direta dos cirurgiões-dentistas, em unidades com ou sem Estratégia de Saúde da Família (ESF), realizam atendimentos da demanda programada, atendimentos de urgência 
(demanda espontânea) e atividades de prevenção em saúde nas escolas pertencentes ao território. Quando as unidades de saúde possuem residente de Odontologia, a experiência do estudante de graduação é ampliada e qualificada, pois o residente realiza ações e desempenha funções diversificadas, inerentes à sua formação em serviço.

O estágio acadêmico é uma oportunidade de revisão, aprimoramento e aprofundamento dos conhecimentos obtidos durante o curso, pois as situações clínicas a que são expostos nas disciplinas e clínicas intramuros são monitoradas pelos professores, que realizam a seleção de pacientes e de procedimentos de forma que o aluno tenha diante de si uma situação de mais fácil controle operatório. Já nos serviços, os pacientes não são escolhidos, todos são atendidos e apresentam situações e necessidades diferenciadas e mais desafiadoras. Os preceptores, quando avaliam o desempenho do estagiário por meio de itens como cumprimento de atividades, iniciativa e criatividade, conhecimento, entre outros (planilha fornecida pelos professores supervisores de estágio), observam que os maiores desafios para os estudantes residem na baixa capacidade de buscar e utilizar alternativas para a solução das necessidades dos pacientes. Estas alternativas não fazem parte do aprendizado no ambiente controlado das clínicas intramuros, pois não experimentam, por exemplo, a falta de algum material restaurador. Neste caso o profissional não deixa de atender seu paciente, buscando outra abordagem clínica no tratamento, como a substituição por um material temporário, até que a situação possa ser resolvida. Um dos depoimentos escritos em relatório ilustra a situação:

"Pude aprender a trabalhar sem alguns materiais e instrumentais. Muitas vezes precisei ser criativo."
Aliado a isto, está o fato de que no serviço público de saúde, especialmente na Estratégia Saúde da Família, as soluções para as necessidades da população extrapolam a Odontologia, sendo que nas IES o foco é basicamente clínico odontológico.

Ao término do estágio os estudantes entregam um relatório, parte da documentação obrigatória prevista no estágio curricular, onde fazem as observações relativas às experiências vividas e o conhecimento adquirido durante o período, com discussão em sala de aula com os professores supervisores. A cada semestre um relatório síntese é elaborado e enviado à Secretaria de Município da Saúde, para ser discutido com os profissionais dos serviços.

A análise destes relatórios permite avaliar que a atividade tem sido bem aceita pela maioria dos estudantes e profissionais de saúde, pois se manifestam com depoimentos como:

"Adquiri mais responsabilidade, aprendizado e autonomia na prática clínica, e ganhei em experiência de vida ao sair da minha zona de conforto $e$ interagir com uma comunidade desconhecida para mim".

Também para os profissionais é um momento de conhecimentos novos por meio da troca de informações com os estudantes. Neste sentido, os preceptores solicitam que a instituição de ensino proporcione atualizações formais e regulares para a equipe de saúde bucal.

Diante disto, alguns professores já ministram, a cada dois meses, cursos de atualização, de temáticas elencadas pelos cirurgiões-dentistas. A metodologia para operacionalizar estas ações é pactuada a cada semestre entre todos os envolvidos (cirurgiõesdentistas, preceptores, professores, estudantes e residentes). Há possibilidade de todos elencarem as temáticas por meio de formulário de sugestões. 
Residência Multiprofissional Integrada em Sistema Público de Saúde - Atenção Básica / Estratégia de Saúde da Família

A experiência da inserção de residentes nas equipes de Estratégia de Saúde da Família tem sido marcada pela indução de mudanças no processo de trabalho. Os residentes surgem como uma equipe de apoio que, além de reconhecer os desafios dos serviços, colaboram para seu enfrentamento, atuando no planejamento, execução e avaliação das ações de saúde. A parceria entre os profissionais da equipe de saúde dos serviços e os profissionais em formação proporciona a ampliação do vínculo e do cuidado da comunidade.

Dentre as ações colocadas em prática em conjunto com a residência estão: o aumento da cobertura da primeira consulta odontológica a crianças de 0 a 2 anos; o acesso ampliado para o agendamento de consultas odontológicas; a implementação da rotina de visitas domiciliares programadas quinzenais a usuários em situação de maior vulnerabilidade; as capacitações sobre saúde bucal para as equipes da ESF; a participação no desenvolvimento do Programa Saúde na Escola (PSE), que tem por objetivo promover ações multidisciplinares no campo da educação em saúde escolar. A participação dos residentes tem sido fundamental na potencialização das ações do PSE, colaborando na construção das matrizes de intervenção, nas atividades de educação e prevenção para crianças e adolescentes e no estreitamento de laços entre a equipe de saúde e os profissionais de educação.

Os residentes também se inserem nos grupos de saúde constituídos nas unidades básicas, que compõem uma das formas de aproximação da equipe de saúde com os usuários. Nos grupos (de crianças, jovens, adultos, idosos e gestantes) são realizados acolhimento e atividades de educação vinculadas ao autocuidado e à autoestima. São utilizadas como estratégias de abordagem as dinâmicas de grupo, o apoio de facilitadores externos convidados para disparar ideias que orientam o início dos encontros, e a troca de experiência entre os participantes por meio de relatos de vivências.

O residente também realiza ações de campo como construção de Projeto Terapêutico Singular (PTS), visitas domiciliares multiprofissionais, planejamento e gerenciamento da unidade e apoio interdisciplinar nas ações dos demais núcleos.

A articulação ensino-serviço por meio da Residência Multiprofissional tem sido o esteio para uma formação mais reflexiva dos profissionais que futuramente atuarão no SUS, assim como para uma permanente renovação e qualificação daqueles que já fazem parte dele. Além disso, tem potencializado o fortalecimento do trabalho em equipe e a integralidade da atenção ao usuário, fugindo da lógica da fragmentação da assistência e proporcionando vivências de aprendizado coletivo.

\section{Residência Multiprofissional em Sistema Público de Saúde - Vigilância em Saúde / Gestão e Planejamento}

A residência na área de Vigilância em Saúde - Gestão e Planejamento envolve a coordenação, planejamento, monitoramento e avaliação das ações em saúde dos 32 municípios que compõem a $4^{\text {a }}$ Coordenadoria Regional de Saúde localizada na região central do estado do Rio Grande do Sul. A articulação dos serviços de saúde, tanto na gestão como na assistência, se dá de forma interdisciplinar, intersetorial e interinstitucional, com base nas políticas nacionais de saúde.

A complementação da formação dos residentes acontece também na Secretaria Municipal da Saúde de Santa Maria, no setor da atenção básica, onde vivenciam a gestão da saúde 
no nível local.

A formação nesta área possibilita $o$ aprendizado em serviço baseado em ações multiprofissionais, relações interpessoais, troca de saberes e transdisciplinaridade durante a construção de fluxos e protocolos, a determinação do percurso transversal da saúde bucal nas políticas de saúde e a análise de indicadores de saúde.

Os residentes na $4^{\mathrm{a}} \mathrm{CRS}$ participam de atividades como: apoio institucional aos tutores municipais na planificação da Atenção Primária à Saúde; reuniões mensais com os coordenadores municipais de saúde bucal; educação continuada das Equipes de Saúde Bucal; construção de fluxos e protocolos com base no Plano Regional de Saúde Bucal, entre outras.

\section{Plano Regional de Saúde Bucal}

Em 2015 iniciou-se a estruturação, pela primeira vez, do Plano Regional de Saúde Bucal da $1^{\text {a }}$ e $2^{\mathrm{a}}$ regiões de saúde do Rio Grande do Sul, Verdes Campos e Entre Rios, que compõem o âmbito de atuação da $4^{\mathrm{a}} \mathrm{CRS}$, ação conduzida pelo coordenador regional de saúde bucal.

A partir de um estudo situacional da saúde bucal na área de abrangência da coordenadoria, vários encontros com os coordenadores de cada município foram organizados, nos quais foram discutidas as necessidades das regiões de saúde e as pactuações necessárias para o planejamento. $\mathrm{O}$ trabalho foi muito bem recebido pelos secretários municipais e proporcionou grande visibilidade à área de saúde bucal da $4^{\mathrm{a}} \mathrm{CRS}$.

O plano foi apresentado e aprovado na Comissão Intergestora Regional e na Comissão Intergestora Bipartite do Rio Grande do Sul sem ressalvas. Esta oportunidade permitiu que os residentes tivessem uma vivência ímpar na sua formação, estimulando-os a participar da elaboração de planos municipais de saúde bucal com elevadas competências e habilidades.

\section{Educação permanente dos trabalhadores de saúde bucal}

Durante as reuniões com os coordenadores de saúde bucal da $4^{\mathrm{a}} \mathrm{CRS}$ constatou-se a necessidade de educação permanente para os profissionais da saúde bucal. Nos encontros ocorre o momento de aperfeiçoamento sobre algum tema relevante e escolhido pelos profissionais, além da reunião de trabalho. Entre os assuntos já abordados estão indicadores de saúde, potencialidades da saúde bucal, sistemas de informação e normatizações do SUS, entre outros.

Dentre as capacitações implementadas está a realização do Maio Vermelho, ação conjunta organizada por entidades de classe, prefeituras municipais, instituições de ensino e Conselho Regional de Odontologia do Rio Grande do Sul, que proporciona aos profissionais relembrar pontos importantes acerca de diagnóstico precoce do câncer bucal, lesões potencialmente malignas, técnica e fluxo da biópsia. Os profissionais são também estimulados a realizar ações de prevenção e promoção à saúde em seus municípios.

Os residentes participaram também das oficinas para a Planificação da Atenção Primária em Saúde, um projeto do CONASS que usa como estratégia a realização de seis oficinas com os trabalhadores - com duração de dois dias cada uma, realizadas mensalmente - criando consensos nos conceitos, definindo prioridades, propostas e ações para a gestão e equipes, reorganizando os serviços e redes de atenção e integrando a atenção ambulatorial e especializada. Entre os temas abordados estão: redes de atenção à saúde, territorialização em saúde, sistemas de informações, assistência farmacêutica, PMAQ, processos de trabalho em UBS. No processo de tutoria da planificação, que ocorre após as oficinas iniciais, foram realizadas 
outras oficinas com temáticas específicas para os profissionais da saúde bucal, e que tiveram a participação de outras categorias profissionais, visando integração dos processos de trabalho. Está sendo construído e implementado um Guia Regional de Saúde Bucal que traz ferramentas para aperfeiçoar o processo de trabalho das equipes.

\section{Plano Municipal de Saúde Bucal de Santa Maria}

O Plano Municipal de Saúde Bucal de Santa Maria, aprovado em 2016, contou com a participação dos residentes na sua elaboração. Várias ações propostas no plano já estão sendo concretizadas, como o projeto Sorria Santa Maria. Nesta ação são proporcionados atendimentos odontológicos e ações de educação e prevenção para organizar a demanda reprimida da saúde bucal na atenção básica e para estabelecer um atendimento odontológico a gestantes, inexistente até então no município. A Sala de Espera Educativa é organizada por uma equipe multiprofissional e interdisciplinar de residentes formada por fonoaudiólogos, farmacêuticos, enfermeiros e nutricionistas, criando um espaço de diálogo sobre saúde e outras temáticas com os usuários que esperam o horário da sua consulta odontológica.

\section{Encontro Regional de Saúde Bucal}

O encontro surgiu da intenção de elaborar um espaço de trocas de experiências e de formação continuada no município. Dada a interação dos residentes com o município e com a $4^{\text {a }}$ CRS, foi percebido que este espaço também era uma necessidade regional.

O I Encontro Regional de Saúde Bucal foi realizado em outubro de 2016 por iniciativa da Coordenação da Política de Saúde Bucal de Santa Maria e pela Coordenação da Política Regional de Saúde Bucal da $4^{a}$ CRS. A participação da
Residência Multiprofissional e o apoio de docentes da UFSM e Universidade Franciscana (UNIFRA) qualificou esta iniciativa. Este encontro teve por objetivo comprometer os profissionais de saúde bucal e gestores na execução das ações e objetivos contidos nos Planos Municipais de Saúde Bucal, além de promover a educação permanente dos trabalhadores de saúde bucal da região. Foi apresentado e discutido o Plano Municipal de Saúde Bucal de Santa Maria e, por meio de mesas redondas e palestras, foram abordados diversos temas elencados previamente pelos profissionais dos serviços que pudessem estimular a atenção e a gestão em saúde.

\section{Potencialidades e limitações da integração ensino-serviço-gestão}

São inegáveis as possibilidades de formação qualificada quando há uma efetiva integração do ambiente formador com a realidade dos serviços de saúde. Elementos teóricos da formação só farão sentido aos estudantes quando forem concretizados no cotidiano da prática em saúde.

A postura crítica, reflexiva e proativa que se deseja de um estudante e de um profissional de saúde pode ser construída conjuntamente, por meio da integração entre instituições de ensino e serviços de saúde, que devem possuir objetivos claros e firmes na busca da resolução das necessidades e qualificação da saúde da população. Esta é, primordialmente, a função destas instituições. Esta construção envolve não somente aspectos formativos técnico-científicos, éticos e humanísticos dos estudantes, mas a transformação do processo de trabalho dos profissionais pela problematização de suas práticas cotidianas ${ }^{8,9}$.

A Residência traz impulsos que faltam para alavancar as mudanças que os serviços necessitam, e da mesma forma o serviço 
proporciona a oportunidade da construção do conhecimento por meio da prática. Os estágios da graduação consolidam a parceria com os cirurgiões-dentistas da rede pública, e estimulam que estes busquem formação continuada.

E uma das mais marcantes potencialidades desta integração reside na inserção dos docentes nos processos de formação e de trabalho proporcionados pela residência e pelos estágios, potencializando e qualificando a prática de ensino e aprendizagem em saúde coletiva.

São muitos os obstáculos e limitações encontrados pelos estudantes e profissionais recém-formados nos serviços, desde aspectos relacionados ao desconhecimento sobre o SUS, passando pela (des) construção do olhar técnicoacadêmico direcionado para uma realidade que era distante na sua formação e chegando ao ponto de reconhecer para si a necessidade de mudar conceitos e posturas e dissolver pré-conceitos. Isto é percebido claramente quando são analisados os depoimentos dos estudantes, tanto aqueles escritos nos relatórios de estágio quanto nos encontros presenciais para discussão dos processos de trabalho:

"Tive que reavaliar meus conceitos."

"Os pacientes precisavam mais do que atenção básica, precisavam de serviços endodônticos, protéticos e até psicológicos."

"A odontologia prestada dependia mais do recurso humano do que de materiais. Estes estavam longe do ideal preconizado pelas escolas de graduação e pósgraduação. A versatilidade do profissional era testada a todo o momento."

"Observei uma organização inesperada."

$\mathrm{O}$ que se aspira é que processos de ensino, de aprendizado e de práticas envolvam todos os sujeitos a um mesmo tempo, lugar e propósitos, capazes de transformar qualitativamente a saúde da população.

\section{CONSIDERAÇÕES FINAIS}

Os locais onde os estudantes e residentes realizam sua formação decorrem de pactuação interinstitucional entre a Universidade e as Secretarias de Município e do Estado. As equipes de saúde participam da construção coletiva desta integração do ensino com o serviço e esta aproximação dos diferentes cenários e profissionais permitiu materializar a integração ensino-serviço-gestão no município de Santa Maria, fortalecendo a cooperação e permitindo a corresponsabilização.

Certamente a instituição de ensino ainda tem um longo caminho a percorrer na consolidação de sua responsabilidade com a comunidade e no atendimento das demandas populacionais no que se refere à formação de um profissional de saúde bucal comprometido com o sistema público de saúde. Mas pode-se inferir a potencialidade desta integração e a sua capacidade de influência para a transformação das práticas e do ensino por meio da ressignificação das relações, e desta com o cuidado em saúde.

\section{ABSTRACT \\ Teaching-service-management integration in oral health in Santa Maria and region: experience report on academic curricular internship and Multiprofessional Residence}

The authors describe the experiences of teachingservice-management integration between the Federal University of Santa Maria, the Municipality of Santa Maria and the 4th Regional Health Coordination of Rio Grande do Sul. The objective is to report the central actions planned and executed by teachers, managers, residents and undergraduate students of these three instances. The involvement of undergraduate students in compulsory traineeship activities is evaluated through the analysis of the reports and the in-service training experiences of residents of 
the Multiprofessional Residency, by recording the activities performed in the public health services of the municipality. We address the advances achieved in the implementation of the actions, the difficulties and potentialities found in their articulation, and emphasize the importance of integration spaces as privileged environments in the continuing training of dental surgeons and trainees. The close relationship between the Multiprofessional Residency in Health and the municipal and regional management allowed for the creation of spaces for dialogue between professionals in training, teachers, managers and users of the service, providing reflection on the process of production of care, co-responsibility for the professional training and the quality of the health service.

Descriptors: Services of Teaching-Assistance Integration. Health Services. Health Services Management.

\section{REFERÊNCIAS}

1. Freitas STF. História social da cárie dentária. Bauru: EDUSC; 2001, 126p.

2. Toassi RFC, Stobäus CD, Mosquera JJM, Moysés SJ. Currículo integrado no ensino de Odontologia: novos sentidos para a formação na área da saúde. Interface Comum Saúde Educ. 2012; 16(41): 529-42.

3. Kovaleski DF, Freitas SFT, Botazzo C. Disciplinarização da boca, a autonomia do indivíduo na sociedade do trabalho. Ciênc Saúde Coletiva. 2006; 11(1):97-103.

4. González, AD. Mudança na formação superior de profissionais de saúde: experiências de ativadores do Paraná. [Dissertação]. Londrina: Universidade Estadual de Londrina; 2008.

5. Morin E. Educação e complexidade: os sete saberes e outros ensaios. Almeida MC,
Carvalho EA. (Org.). 4 ed. São Paulo: Cortez, 2007.

6. Albuquerque VS, Gomes AP, Rezende CHA, Sampaio MX, Dias OV, Lugarinho RM. A integração ensino-serviço no contexto dos processos de mudanças na formação superior dos profissionais da saúde. Rev Bras Educ Méd. 2008;32(3): 356-62.

7. Zarpelon LFB, Batista NA, Terencio ML. Integração ensino-serviço no contexto das escolas médicas brasileiras: revisão integrativa. Ciênc Saúde Coletiva. 2017. [Acesso em 25 set. 2017]. Disponível em: http://www.cienciaesaudecoletiva.com.br/ar tigos/integracao-ensinoservico-no-contextodas-escolas-medicas-brasileiras-revisaointegrativa/16087

8. Lima LL, Jorge MSB, Gomes ILV, Morais JB. Sentidos e significados da integralidade e interdisciplinaridade na formação em saúde. In: Jorge MSB, Lima LL, Pinto AGA (Orgs). Caminhos da formação em saúde: políticas, desafios e contradições - ensino, pesquisa, cuidado e gestão. Fortaleza: EdUECE; 2012.

9. Ceccim RB, Feuerwerker LCM. O quadrilátero da formação para a área da saúde: ensino, gestão, atenção e controle Social. Physis. 2004;14(1):41-65.

Correspondência para:

Jessye Melgarejo do Amaral Giordani

E-mail: jessyesm@hotmail.com

Departamento de Estomatologia

Rua Marechal Floriano Peixoto, 1184/sala 109

97015-372 Centro - Santa Maria/RS 